\title{
Detection of Class 1,2, and 3 Integrons Among Klebsiella pneumoniae Isolated from Children in Tehran Hospitals
}

\author{
Safoura Derakhshan ${ }^{1}$, Shahin Najar Peerayeh ${ }^{1,}$, Fatemeh Fallah ${ }^{2}$, Bita Bakhshi ${ }^{1}$, Moham- \\ mad Rahbar ${ }^{3}$, Abbas Ashrafi ${ }^{4}$ \\ ${ }^{1}$ Department of Bacteriology, Faculty of Medical Sciences, Tarbiat Modares University, Tehran, IR Iran \\ 2 Pediatric Infections Research Center, Shahid Beheshti University of Medical Sciences, Tehran, IR Iran \\ 3 Department of Microbiology, Iranian Reference Health Laboratory, Ministry of Health and Medical Education, Tehran, IR Iran \\ 4 Department of Pathology, Imam Khomeini Hospitals' complex, Tehran, IR Iran \\ *Corresponding author: Shahin Najar Peerayeh, Department of Bacteriology, Faculty of Medical Sciences, Tarbiat Modares University, P.o.Box: 14115-158, Tehran, IR Iran. Tel: +98- \\ 2182883870, Fax:+98-2182884555, E-mail: najarp_s@modares.ac.ir.
}

.Received: April 29, 2013; Revised: Jun 08, 2013; Accepted: Jun 18, 2013

Background: CTX-M-type $\beta$-lactamases are increasingly becoming the predominant ESBLs globally in recent years. Integrons are genetic elements which can integrate gene cassettes, usually antibiotic resistance genes.

Objectives: The aims were to determine antibiotic susceptibility and to detect genes encoding CTX-M-1 group enzymes and class 1,2, and 3 integrons among the Klebsiella pneumoniae isolated from children in Tehran hospitals, Iran.

Patients and Methods: Thirty-one K. pneumoniae isolates were collected from samples of children aged 0-12 years admitted to three hospitals in Tehran between May and December 2011, and identified using biochemical tests and PCR. Susceptibility of isolates to 14 antibiotic disks was determined using disk diffusion method. The combined disk method was used for the detection of ESBL. The presence of bla $_{\text {CTX-M-1 }}$ group and class 1, 2, and 3 integrons was investigated by PCR.

Results: Most of the isolates showed high level of resistance: 17 isolates were simultaneously resistant to Amoxicillin-Clavulanic acid, Cefotaxime, Ceftriaxone, Aztreonam, and Ceftazidime (17/31, 54.9\%). All were susceptible to Imipenem and Ciprofloxacin. ESBL production was detected in $54.9 \%(17 / 31)$. The $b l a_{\text {CIX-M-1 }}$ group was detected in all Cefotaxime-resistant isolates $(17 / 31,54.9 \%)$. Class 1 integron was detected in 8 isolates (25.8\%). The class 2 and 3 integrons were not detected.

Conclusions: The results showed that the CTX-M-1- producing K. pneumoniae is already present in some parts of Tehran. The presence of class I integron genes among resistant strains of K. pneumoniae highlights the continued monitoring of drug resistance in clinical settings.

Keywords: Klebsiella pneumoniae; Drug Resistance; CTX-M Beta-lactamase; Integrons

\section{Background}

Extended-spectrum $\beta$-lactamases (ESBLs) have been observed in virtually all species of the family Enterobacteriaceae mainly Escherichia coli and Klebsiella pneumoniae (1). Among ESBLs the most widespread and clinically relevant are class A ESBLs of TEM, SHV, and CTX-M types. CTX-M-type $\beta$-lactamases are increasingly becoming the predominant ESBLs globally in recent years, including Asia (2). These enzymes have been classified into five major groups by amino acid sequence similarities: clusters of CTX-M-1, CTX-M-2, CTX-M-8, CTX-M-9, and CTX-M-25/26. They have a preferential hydrolysis of Cefotaxime over Ceftazidime; although some CTX-M type ESBLs, including CTX-M-15, show good activity against Ceftazidime (3).
Recent advances in the molecular characterization of antibiotic resistance mechanisms have resulted in the discovery of genetic elements, called integrons which can integrate antibiotic resistance genes. Several studies have reported integron distributions in multidrug resistant strains isolated from animals and humans. The role of integrons in the development of multiple resistances relies on their unique capacity to acquire gene cassettes and express cassette-associated genes (4). An integron, which can be located either on the bacterial chromosome or on a plasmid, includes the gene for an integrase site (int) and for an adjacent recombination site (attI). So far three classes of antibiotic-resistance-encoding integrons have been identified. Each class has its own integrase. Among the antibiotic-resistance integrons, class

Implication for health policy/practice/research/medical education:

Extended-spectrum $\beta$-lactamase (ESBL)-producing Klebsiella pneumoniae have rapidly spread worldwide. CTX-M-type $\beta$-lactamases are increasingly becoming the predominant ESBLs globally in recent years, including Asia. The role of integrons in the development of multiple resistance threats the world. Thus, the aim of our study was to detect the genes encoding CTX-M-1 group enzymes and class 1, 2, and 3 integrons among the K. pneumoniae isolated from children in Tehran hospitals, Iran.

Copyright (C 2014, Pediartric Infections Research Center. This is an Open Access article distributed under the terms of the Creative Commons Attribution License (http://creativecommons.org/licenses/by/3.0), which permits unrestricted use, distribution, and reproduction in any medium, provided the original work is properly cited. 
1 integrons are the most common integron type, class 2 integrons are embedded in Tn7-family transposons and only one example of a class 3 integron is known (5).

\section{Objectives}

The aim of this study was to investigate the antimicrobial susceptibility and to determine the prevalence of CTXM-1 group enzymes and class 1, 2, and 3 integrons genes among K. pneumoniae isolated from children in Tehran hospitals, Iran.

\section{Patients and Methods}

\subsection{Bacterial Isolates and Identification}

A total of 31 nonduplicate Klebsiella pneumoniae isolates were collected from clinical specimens of children aged 0-12 years admitted to three hospitals in Tehran (LoghmanE Hakim in the southwest, Imam Khomeini in the center, and Milad in the northwest) between May and December 2011. They were identified as K. pneumoniae using biochemical tests and a PCR method to confirm the identification of K. pneumoniae subsp. pneumoniae, using published specific primer pair for K. pneumoniae 16S-23S internal transcribed spacer gene (6): Pf: $5^{\prime}$-ATTTGAAGAGGTTGCAAACGAT-3' and Pr1: 5'-TTCACTCTGAAGTTTTCTTGTGTTC-3' (amplicon size: $130 \mathrm{bp}$ ). Genomic DNA was prepared by boiling the isolates and used as the templates (7). Cycling conditions were as follows: initial denaturation at $94^{\circ} \mathrm{C}$ for $5 \mathrm{~min} ; 35$ cycles of $94^{\circ} \mathrm{C}$ for $1 \mathrm{~min}, 58^{\circ} \mathrm{C}$ for $1 \mathrm{~min}$, and $72^{\circ} \mathrm{C}$ for $1 \mathrm{~min}$ followed by a final elongation at $72^{\circ} \mathrm{C}$ for $7 \mathrm{~min}$. K. pneumoniae ATCC13883 was used as positive control.

\subsection{Antibiotic Susceptibility Testing}

The antibiotic susceptibilities were determined by disk diffusion method on Mueller-Hinton agar plates (Merck, Darmstadt, Germany) as recommended by the clinical and laboratory standards institute (CLSI) (8). The disks containing the following antibiotics $(\mu \mathrm{g})$ were used (Mast, UK): Cefotaxime (CTX, 30), Ceftriaxone (CRO, 30), Ceftazidime (CAZ, 30), Imipenem (IPM, 10), AmoxicillinClavulanic acid (AUG, 30), Aztreonam (ATM, 30), Ciprofloxacin (CIP, 5), Tobramycin (TN, 10), Tetracycline (T, 30), Trimethoprim-sulfamethoxazole (TS, 25), Gentamicin (GM, 10), Cefepime (CPM, 30), Cefoxitin (FOX, 30), and Amikacin (AK, 30). E. coli ATCC 25922 was used as quality control for antimicrobial susceptibility.

\subsection{ESBL Screening and Confirmation by Pheno- typic Methods}

The isolates showing reduced susceptibility to Ceftazidime or Cefotaxime or both were tested for ESBLs production by the combined disk method according to the CLSI guidelines (8). The inoculum and incubation conditions were the same as for standard disk diffusion recommendations. Combined disk method was performed using four disks ( $\mu \mathrm{g})$ : Cefotaxime (CTX) (30), Cefotaxime + Clavulanic acid (10), Ceftazidime (CAZ) (30), and Ceftazidime + Clavulanic acid (10). A $\geq 5 \mathrm{~mm}$ increase in zone diameter for either antimicrobial agent tested in combined with Clavulanic acid versus its zone when tested alone was designated as ESBL positive (8). Quality control for the production of ESBLs was performed using E. coli ATCC 25922 and K. pneumoniae ATCC 700603 as negative and positive controls, respectively.

\subsection{CTX-M-1 $\beta$-lactamase and Class 1,2 , and 3 Inte- grons Identification}

As mentioned above, genomic DNA was prepared by boiling the isolates and used as the templates for PCR reactions (7). Amplification of all genes was performed on Gene Amp PCR System PTC-1148 (Bio-Rad, Foster City, CA, USA).

For detection of $b l a_{\mathrm{CTX}-\mathrm{M}-1}$ ESBL genes, PCR was performed using primers for bla ${ }_{\mathrm{CTX}-\mathrm{M}-1}$ group genes: $5^{-}$-GGTTAAAAAATCACTGCGTC-3' and 5 ${ }^{\prime}$-TTGGTGACGATTTTAGCCGC-3' (amplicon size: $864 \mathrm{bp}$ ) (9). Amplification of class 1, 2, and 3 integrons was performed for bla $a_{\text {CTX-M-1 }}$ positive isolates using published specific primer pairs for intI1, intI2, and intI3 genes as follows: $5^{-}$-CAGTGGACATAAGCCTGTTC-3 and $5^{-}$-CCCGAGGCATAGACTGTA-3' (for intI1, amplicon size: 160 bp) (10), $5^{\prime}$-CACGGATATGCGACAAAAAGGT-3' and $5^{\prime}$-GTAGCAAACGAGTGACGAAATG-3'(for intI2, amplicon size: 789 bp) (11), and $5^{-}$-GCCTCCGGCAGCGACTTTCAG-3' and $5^{\prime}$-ACGGATCTGCCAAACCTGACT-3' for intI3, amplicon size: $979 \mathrm{bp})(11)$. Multiplex PCR was used to detect intI2 and intI3 genes.

Cycling conditions were as follows: Initial denaturation at $94^{\circ} \mathrm{C}$ for $5 \mathrm{~min}$; 35 cycles of $94^{\circ} \mathrm{C}$ for $1 \mathrm{~min}, 54^{\circ} \mathrm{C}$ for 1 $\min$, and $72^{\circ} \mathrm{C}$ for $1 \mathrm{~min}$ followed by a final elongation at $72^{\circ} \mathrm{C}$ for $7 \mathrm{~min}$. Conditions were the same for all genes except for intI2 and intI3 multiplex-PCR for which the annealing temperature was $60^{\circ} \mathrm{C}$. The PCR products were analyzed by electrophoresis with $1 \%$ agarose gels in $1 \mathrm{X}$ TAE (Tris-Acetate-EDTA) buffer. The gels were stained with $5 \mu \mathrm{g} / \mathrm{mL}$ ethidium bromide, and the PCR products were visualized under the UV light.

\section{Results}

All K. pneumoniae isolates with positive findings for biochemical tests also showed positive PCR results for the 16S-23S internal transcribed spacer region. Of 31 isolates, 21 were isolated from urine (67.7\%), 2 from wound, 2 from tracheal secretions, and 6 from other samples (including catheter, eye, and etc.). 24 strains were isolated from females $(24 / 31,77.4 \%)$. Eleven strains were isolated from $\operatorname{NICU}(35.5 \%), 11$ from pediatric (35.5\%), and 6 from Neonatal wards (19.3\%) (Table 1). Analysis of the antimicrobial susceptibility profile of 
Derakhshan S et al.

\begin{tabular}{|c|c|c|c|c|}
\hline Strains & Hospital & Sex & Ward & Specimen \\
\hline 1 & Milad & $\mathrm{F}^{\mathrm{a}}$ & $\mathrm{NICU}^{\mathrm{a}}$ & Urine \\
\hline 2 & Milad & $\mathrm{M}^{\mathrm{a}}$ & Pediatric & Urine \\
\hline 3 & Milad & $\mathrm{F}$ & NICU & Urine \\
\hline 4 & Milad & $\mathrm{F}$ & Pediatric & Urine \\
\hline 5 & Milad & $\mathrm{F}$ & Neonatal & Urine \\
\hline 6 & Milad & $\mathrm{F}$ & Neonatal & Urine \\
\hline 7 & Milad & $\mathrm{F}$ & NICU & Urine \\
\hline 8 & Milad & $\mathrm{F}$ & NICU & Urine \\
\hline 9 & Milad & $\mathrm{F}$ & Pediatric & Urine \\
\hline 10 & Loqhman & M & Pediatric & Wound \\
\hline 11 & Milad & M & NICU & Peritoneal secretions \\
\hline 12 & Milad & $\mathrm{F}$ & Pediatric & Urine \\
\hline 13 & Milad & M & Pediatric & Urine \\
\hline 14 & Milad & $\mathrm{F}$ & NICU & Eye \\
\hline 15 & Milad & $\mathrm{F}$ & NICU & Urine \\
\hline 16 & Milad & $\mathrm{F}$ & NICU & Tracheal secretions \\
\hline 17 & Milad & $\mathrm{F}$ & Pediatric & Urine \\
\hline 18 & Imam & M & Pediatric & Urine \\
\hline 19 & Imam & $\mathrm{F}$ & NICU & Eye \\
\hline 20 & Imam & $\mathrm{F}$ & Neonatal & Urine \\
\hline 21 & Imam & $\mathrm{F}$ & NICU & Tracheal secretions \\
\hline 22 & Imam & $\mathrm{F}$ & Neonatal & Umbilical secretions \\
\hline 23 & Imam & $\mathrm{F}$ & Neonatal & Urine \\
\hline 24 & Imam & M & Pediatric & Urine \\
\hline 25 & Imam & $\mathrm{F}$ & Neonatal & Urine \\
\hline 26 & Milad & M & PICU $^{\mathrm{a}}$ & Catheter \\
\hline 27 & Milad & $\mathrm{F}$ & NICU & Urine \\
\hline 28 & Milad & $\mathrm{F}$ & Emergency & Urine \\
\hline 29 & Milad & $\mathrm{F}$ & Pediatric & Wound \\
\hline 30 & Loqhman & $\mathrm{F}$ & Pediatric & Urine \\
\hline 31 & Milad & $\mathrm{F}$ & PICU & Throat secretions \\
\hline
\end{tabular}

a Abbreviations: F, female; M, male; NICU, neonatal intensive care unit; PICU, pediatric intensive care unit

the isolates showed that all were susceptible to Imipenem and Ciprofloxacin. Of 31 isolates, $54.9 \%$ were simultaneously resistant to Amoxicillin-Clavulanic acid, Cefotaxime, Ceftriaxone, Aztreonam, and Ceftazidime ( $\mathrm{n}=$ 17), 16 isolates were resistant to Tobramycin, Cefepime, and Amikacin (51.6\%), 14 isolates were resistant to gentamicin (45.2\%), 3 isolates were resistant to TrimethoprimSulfamethoxazole, and Tetracycline (9.67\%), and 1 isolate was resistant to Cefoxitin (3.2\%). Among 31 isolates, 17 (54.9\%) were identified as ESBLs producers according to the combined disk method. ESBLs-producer isolates demonstrated a higher degree of multidrug resistance as compared to non-ESBLs (Figure 1 ) (P value $<0.05$ ). The bla $a_{\text {CTX-M-1 }}$ group gene was detected in all cefotaxime-resistant isolates (17/31, 54.9\%) (Figure 2).

Using the PCR assay, 8 (25.8\%) of 31 isolates were found to have positive results for the presence of class 1 integrons (Figure 3 ). Five strains were isolated from urine $(5 / 8,62.5 \%)$ and 7 were isolated from females $(7 / 8,87.5 \%)$. Two strains were isolated from NICU (2/8, 25\%), 2 from neonatal (2/8, 25\%), 3 from Pediatric (3/8, 37.5\%), and 1 from PICU ward. No integrons were found in the remaining isolates. No class 2 or class 3 integrons were detected among isolates. 


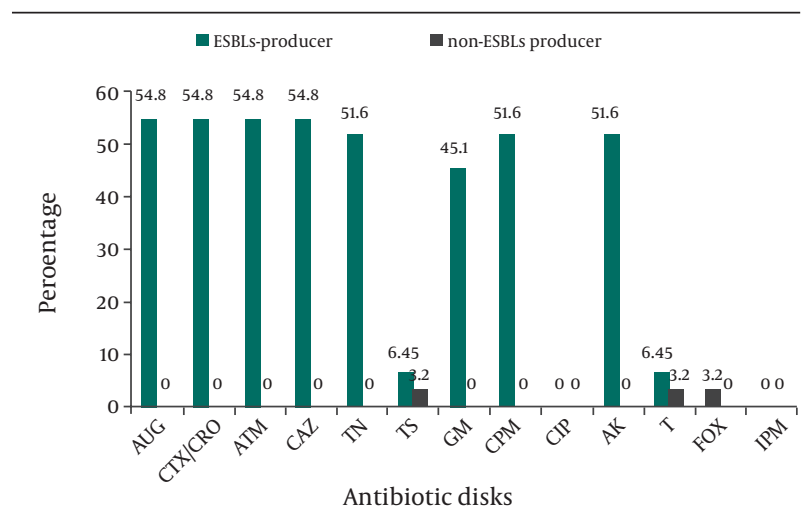

Figure 1. Comparison of Resistance (\%) to Antibiotic Disks in ESBLs Producing and non-ESBLs Producing Isolates of Klebsiella pneumoniae

Figure 2. PCR for Detection of $b l a_{\mathrm{CTX}-\mathrm{M}-1}$ Genes.

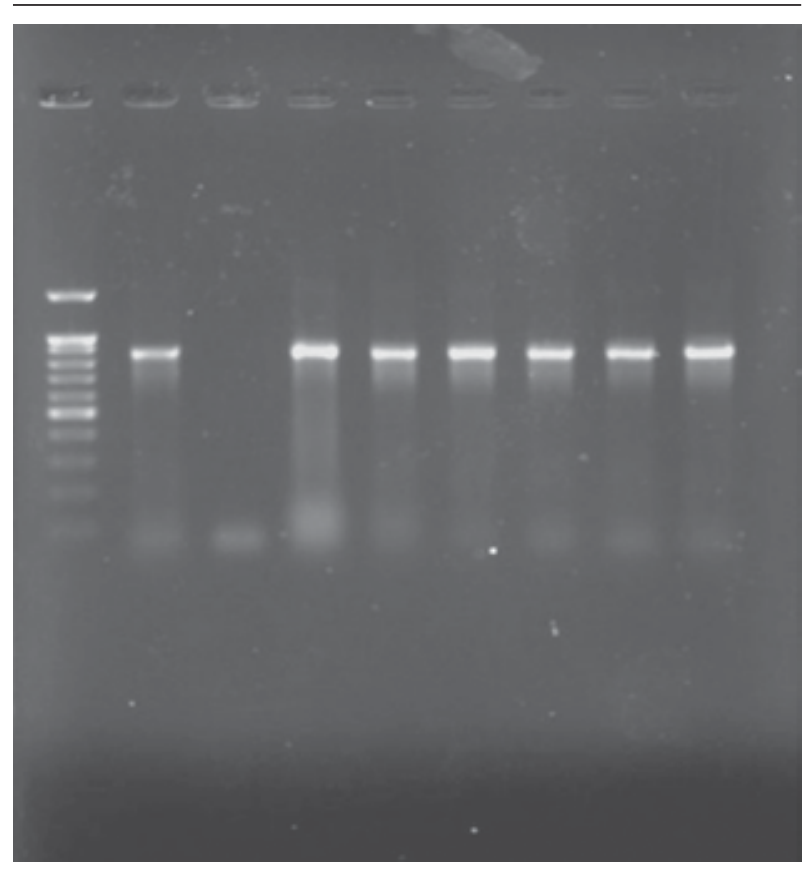

Ladder: 100 bp ladder; 1: positive control; 2: negative control; 3-8: CTX-M group I

\section{Discussion}

Infections caused by ESBL-producing bacteria are increasing in many countries including Iran $(12,13)$. During the last decades, the emergence of antimicrobial drugresistant strains has been reported in K. pneumoniae isolated from community and hospital acquired infections (14-16). Resistance to antimicrobial agents is often associated with the spread of transmissible plasmids and integrons which can be located on the chromosome or plasmids. The ability of integrons to integrate resistance gene cassettes makes them prime pools for the further dissem- ination of antibiotic resistance among clinical isolates of gram-negative bacteria, including K. pneumoniae (17).

Figure 3. PCR for Detection of Class 1 Integron Genes Among Klebsiella Pneumoniae Isolates.

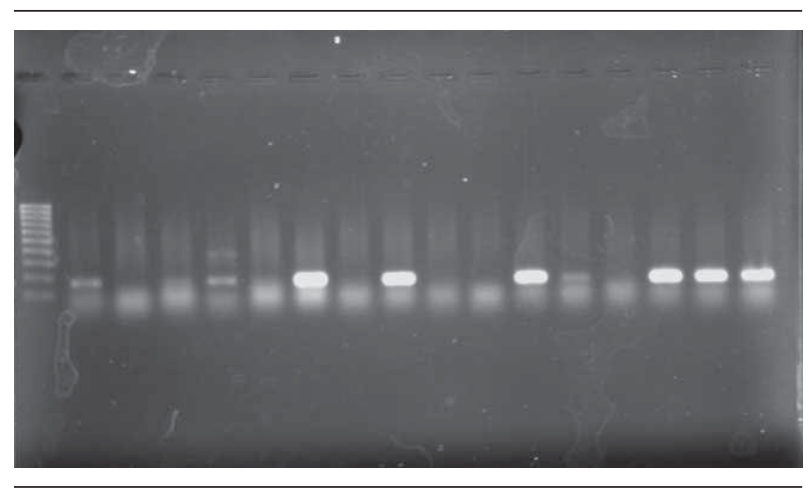

Ladder: 100 bp ladder; 1: positive control; 2: negative control; 3-11: clinical isolates

In this study, the frequency of ESBL-producing K. pneumoniae isolated from children was $54.9 \%$ (17/31). The bla $_{\text {CTX-M-1 }}$-group gene was detected in $54.9 \%$ of isolates. In a study performed by Karimi et al., 19 isolates from $50 \mathrm{~K}$. pneumoniae isolated from children with urinary tract infections (UTI) (38\%), had positive results for ESBLs production (18). In another study performed by Seyed Javadi et al., Of $30 \mathrm{~K}$. pneumoniae isolated from children with UTI, 21 (70\%) were multidrug resistant (19). In a study performed by Feizabadi et al., the prevalence of bla $a_{\text {CTX-M-1 }}$ genes among the K. pneumoniae isolates was $45.2 \%$ (13). Several reports have confirmed the emergence of CTX-M-1-producing $K$. pneumoniae isolates in Sweden, France, Madagascar, and Croatia. Also in Asian countries, CTX-M-1-producing $K$. pneumoniae isolates have been reported from India, Kuwait, Saudi Arabia, Malaysia, The Philippines, Singapore, and Thailand $(2,20,21)$. Various factors involved in these differences including strains isolated from hospitalized patient or from outpatient, the geographic differences across the world, and etc.

In our study, $25.8 \%$ of isolates were found to havepositive results for the presence of class 1 integrons. No class 2 or class 3 integrons were detected. Karimi et al., reported that the prevalence of class 1 integrons was $48 \%$ in $K$. pneumoniae isolates (18) and Seyed Javadi et al., reported that class 1 integrons were found in $13.3 \%$ of the K. pneumoniae isolates (19). In Seyed Javadi study, class 2 integron was not found which is in accordance with our study. In a survey in Taiwan on the prevalence of class 1 integrons in clinical K. pneumoniae isolates collected from Taiwan, during 2 periods (1993 and 2004), class 1 integrons were present in 78 isolates (34.2\%) from 1993 and 129 (32.9\%) from 2004 (22). The most commonly encountered integrons are those of class 1 which may be located on transmissible plasmids and transposons (23).

In conclusion, the results of our study revealed that CTX- 
M-1- producing K. pneumoniae is already present in some parts of Tehran. K. pneumoniae is a very efficient hospital pathogen and, in addition, can readily acquire plasmidmediated CTX-M-1 genes from E. coli. High rates of antimicrobial resistance and the role of integrons in the acquisition and dissemination of resistance genes among bacteria suggest to monitor mechanisms of antimicrobial resistance and to emphasize on the rational use of antimicrobials to decrease the spread of ESBL producing bacteria.

\section{Acknowledgements}

We are grateful to the staff of the microbiology laboratory at the Loghman-E Hakim, Imam Khomeini, and Milad hospital for collecting K. pneumoniae isolates used in this study.

\section{Authors' Contribution}

S. Derakhshan performed the microbiological and molecular studies. S. Najar Peerayeh designed the search. F. Fallah and B. Bakhsi advised the search. M. Rahbar and A. Ashrafi collected the strains. All authors approved the final manuscript.

\section{Financial Disclosure}

This study was performed by a grant from Tarbiat Modares University, Faculty of Medical Sciences, Tehran, Iran, and Pediatric Infections Research Center, Tehran, Iran.

\section{Funding/Support}

There is no funding.

\section{References}

1. Arpin C, Dubois V, Coulange L, Andre C, Fischer I, Noury P, et al. Extended-spectrum beta-lactamase-producing Enterobacteriaceae in community and private health care centers. Antimicrob Agents Chemother. 2003;47(11):3506-14.

2. Lee MY, Ko KS, Kang CI, Chung DR, Peck KR, Song JH. High prevalence of CTX-M-15-producing Klebsiella pneumoniae isolates in Asian countries: diverse clones and clonal dissemination. Int $J$ Antimicrob Agents. 2011;38(2):160-3.

3. Rotimi VO, Jamal W, Pal T, Sovenned A, Albert MJ. Emergence of CTX-M-15 type extended-spectrum beta-lactamase-producing Salmonella spp. in Kuwait and the United Arab Emirates. J Med Microbiol. 2008;57(Pt 7):881-6.

4. Carattoli A. Importance of integrons in the diffusion of resistance. Vet Res. 2001;32(3-4):243-59.

5. Fluit AC, Schmitz FJ. Class 1 integrons, gene cassettes, mobility, and epidemiology. Eur J Clin Microbiol Infect Dis. 1999;18(11):761-70.

6. Turton JF, Perry C, Elgohari S, Hampton CV. PCR characterization and typing of Klebsiella pneumoniae using capsular type-specific, variable number tandem repeat and virulence gene targets. $J$ Med Microbiol. 2010;59(Pt 5):541-7.

7. Doi Y, Adams-Haduch JM, Shivannavar CT, Paterson DL, Gaddad
SM. Faecal carriage of CTX-M-15-producing Klebsiella pneumoniae in patients with acute gastroenteritis. Indian J Med Res. 2009;129(5):599-602.

8. Clinical and Laboratory Standards Institute. Performance standards for antimicrobial susceptibility testing, 20th informational supplement (M100-S20). Vol. 30. Clinical and Laboratory Standards Institute: Wayne Pa. 2011.

9. Li G, Wei Q, Wang Y, Du X, Zhao Y, Jiang X. Novel genetic environment of the plasmid-mediated KPC-3 gene detected in Escherichia coli and Citrobacter freundii isolates from China. Eur J Clin Microbiol Infect Dis. 2011;30(4):575-80.

10. Fiett J, Baraniak A, Mrowka A, Fleischer M, Drulis-Kawa Z, Naumiuk L, et al. Molecular epidemiology of acquired-metallo-betalactamase-producing bacteria in Poland. Antimicrob Agents Chemother. 2006;50(3):880-6.

11. Wen X, Wu Y, Bian F, Sun Y, Zheng X, Zhang Y, et al. High prevalence of atypical class 1 integrons and class 2 integrons in multidrug resistance Shigella flexneri isolated from China. Afr J Microbiol Res. 2012;6(42):6987-93.

12. Mirzaee M, Owlia P, Mansouri S. Distribution of CTX-M $\beta$-lactamase genes among Escherichia coli strains isolated from patients in Iran. Lab Med. 2009;40(12):724-7.

13. Feizabadi MM, Mahamadi-Yeganeh S, Mirsalehian A, Mirafshar SM, Mahboobi M, Nili F, et al. Genetic characterization of ESBL producing strains of Klebsiella pneumoniae from Tehran hospitals. J Infect Dev Ctries. 2010;4(10):609-15.

14. Bhattacharjee A, Sen MR, Prakash P, Gaur A, Anupurba S, Nath G. Observation on integron carriage among clinical isolates of Klebsiella pneumoniae producing extended-spectrum beta-lactamases. Indian J Med Microbiol. 2010;28(3):207-10.

15. Tawfik AF, Alswailem AM, Shibl AM, Al-Agamy MH. Prevalence and genetic characteristics of TEM, SHV, and CTX-M in clinical Klebsiella pneumoniae isolates from Saudi Arabia. Microb Drug Resist. 2011;17(3):383-8.

16. Turner PJ. Extended-spectrum beta-lactamases. Clin Infect Dis. 2005;41 Suppl 4:S273-5.

17. Ranjbar R, Giammanco GM, Farshad S, Owlia P, Aleo A, Mammina C. Serotypes, antibiotic resistance, and class 1 integrons in Salmonella isolates from pediatric cases of enteritis in Tehran, Iran. Foodborne Pathog Dis. 2011;8 (4):547-53.

18. Karimi A, Malekan M, Rahbar M, Navidinia M, Fallah F, Akhoundtabar L. Detection of integron elements and gene groups encoding ESBLs and their prevalence in E.coli and Klebsiella isolated from urine samples by PCR method. 15th International Congress on Infectious Diseases. Thailand. 2012.

19. Seyedjavadi S, Eslami G, Goudarzi M, Goudarzi H, Fallah F. Integrons and multidrug resistance among E. coli and Klebsiella pneumoniae isolated from children with urinary tract infections. Health Med. 2013;7(1):p243.

20. Al Sweih N, Salama MF, Jamal W, Al Hashem G, Rotimi VO. An outbreak of CTX-M-15-producing Klebsiella pneumoniae isolates in an intensive care unit of a teaching hospital in Kuwait. Indian J Med Microbiol. 2011;29(2):130-5.

21. Rubtsova M Yu, Ulyashova MM, Bachmann TT, Schmid RD, Egorov AM. Multiparametric determination of genes and their point mutations for identification of beta-lactamases. Biochemistry (Mosc). 2010;75:1628-49.

22. Chang CY, Fang YT, Tsai SM, Chang LL, Yu WL. Characterization of class 1 integrons and gene cassettes in clinical isolates of Klebsiella pneumoniae from Taiwan. Diagn Microbiol Infect Dis. 2009;65(2):214-6.

23. Gu B, Tong M, Zhao W, Liu G, Ning M, Pan S. Prevalence and characterization of class I integrons among Pseudomonas aeruginosa and Acinetobacter baumannii isolates from patients in Nanjing, China. J Clin Microbiol. 2007;45(1):241-3. 